\title{
Facile fabrication and tribological properties of self-lubricating polyurethane materials with sponge-like structure
}

\author{
B. P. Yang ${ }^{1}$, J. B. Cui ${ }^{1}$, B. Mu ${ }^{1 *}$, J. F. Cui ${ }^{1}$, J. H. Guo ${ }^{1}$, X. Wang $^{2}$ \\ ${ }^{1}$ College of Petrochemical Technology, Lanzhou University of Technology, 730050 Lanzhou, People's Republic of China \\ ${ }^{2}$ School of Chemical Engineering, Northwest University for Nationalities, 730030 Lanzhou, People's Republic of China
}

Received 21 January 2019; accepted in revised form 2 April 2019

\begin{abstract}
Inspired by the lubrication mechanism of human articular cartilage tissue, the porous self-lubricating polyurethane (PU) materials with micron-meter pore were fabricated by non-solvent induced phase separation (NIPS) method with $60 \mathrm{wt} \%$ $\mathrm{N}, \mathrm{N}$-dimethylformamide (DMF) as a coagulation agent. The scanning electron microscopic (SEM) images demonstrated that the cross-section morphology of the materials is similar to that of the sponge and adjacent macropores were connected by interpenetrating pores to exhibit an 'ink-bottle' type pore structure. Meanwhile, the porosity of the porous self-lubricating PU materials could be controlled by adjusting the PU solution concentrations and the oil content increased as the increasing of porosity. The results of centrifugation/heating test indicated that the porous self-lubricating PU materials have an excellent oil retention performance owing to the pore structure. The tribological properties of porous self-lubricating materials with different porosity under various loads and sliding velocities were investigated by a block-on-ring wear tester which revealed that excellent friction reduction and wear resistance properties of the porous self-lubricating materials were achieved by the lubricating oil squeezed from pores which the friction coefficient of the materials significantly reduced, from 0.174 to 0.078 with the increases of oil content. Furthermore, the lubrication mechanism of porous self-lubricating PU material was discussed as well.
\end{abstract}

Keywords: material testing, nonsolvent-induced phase separation (NIPS), porous self-lubricating materials, wear and friction

\section{Introduction}

Friction and wear are unavoidable in the operation of mechanical equipment, which subsequently lead to damages to the engineering facilities. When the bearing is circulated under dry friction, the direct contact of the friction couple causes severe wear and may even result in the bearing seizure [1]. Therefore, oil lubrication with the formation of lubricating film possessing bearing capacity and low tangential shear strength between friction surfaces, could achieve the purpose of reducing friction and anti-wear. With the upgrading of the mechanical devices, the lubrication dilemma engendered by the more and more harsh working conditions, e.g., high-vacuum space circumstance, caused the rising difficulty of external oil supply to the friction interface and hence oil- containing self-lubricating materials commences to take the prominent position. $\mathrm{Mu}$ et al. [2] prepared PU composites filled with microcapsules containing methyl silicone oil as core materials and modified by short carbon fiber. In the friction process, the lubricating oil was released after the destruction of the oilcontaining microcapsule. Thereby the friction and wear properties of PU composites were greatly improved. Yang et al. [3] fabricated PU composite coating by incorporating the silica gel shell microcapsule

${ }^{*}$ Corresponding author, e-mail: mu-bo@163.com

(C) BME-PT 
containing ionic liquid into the PU matrix. The results showed that the ionic liquid released from the broken microcapsule which can effectively lubricate the rubbing surfaces which lead to the considerably reduce of the friction coefficient. However, before the lubricating oil was released and function as a friction reduction additive, the microcapsule loaded materials must be subjected to a certain degree of wear which limits its tribological application to some extent. Therefore, Wang et al. [4] used the cold pressing and sintering processes to prepare porous polyimide with different porosity, which is adopted as self-lubrication retainers to improve the tribological performance of rolling bearings under starved lubrication conditions. Wang et al. [5] prepared a porous self-lubricating PEEK composite with nano/micro multilevel pore structure by molding-leaching and vacuum suction technologies. The porous material is capable of inhaling and storing lubricating oil because of its internal pore structure, if needed, the stored oil was released continuously to the friction surface [6]. Based on these characteristics, porous materials containing oil have been widely employed to avoid the lubrication deficiency status. From the bionics point of view, the surface of the animal joint possesses a layer of porous cartilage tissue for storing lubricating fluid. As the cartilage tissue is subjected to external loads, the lubricant is delivered to maintain the boundary lubrication $[7,8]$.

Recent years, the preparation of porous film has been reported in many different routes. In the discussions of Wang et al [9], controllable polyimide (PI) films with an ordered surface were prepared using silica microspheres as hard template. Chang et al. [10] fabricated the double shell hollow spheres with $\mathrm{TiO}_{2}$ shell encapsulated the hollow acrylic/styrene spheres, and polymeric hollow spheres were dissolved in the polyamic acid solutions to obtain the porous polyimide films which is distinct from normal routine of template removal process with enthetic solvent introduced. Meanwhile, Lu et al. [11] synthesized Nafion membranes with ordered mesoporous structures via a soft micelle template method. Compared with hard template method, the soft template embedded in the matrix can be removed by reflux with hot water instead of toxic chemicals (hydrofluoric acid), forming highly ordered mesoporous structures in membranes. Recent years, non-solvent induced phase separation
(NIPS) method has been applied in the fabrication of porous materials. Ye et al. [12] fabricated porous film with regular micropores by casting polymer solution onto a glass plate and using water droplets generated by an ultrasonic atomizer as the coagulation bath. Xing et al. [13] prepared a poly (L-lactic acid) porous membrane by NIPS, and found that the ultimate structure and properties of the membranes can be controlled by varying the coagulation bath compositions. Compared with the preparation route mentioned above, the significant advantage of the non-solvent induced phase separation (NIPS) lies in the simplicity of fabrication process in which the homogeneous polymer solution is immersed in a coagulation bath to induce phase separation. With the exchange of non-solvent and solvent, the mixture was separated into rich polymer phase and poor polymer phase, followed by phase separation until the rich polymer phase was solidified into membrane [14]. The widespread application of this porous structure was focused on wastewater treatment $[15,16]$, lithium ion battery separators [17], vapor sensors [18], supercapacitors [19] and three-dimensional scaffolds [20]. Inspired by the lubrication mechanism of joint lubrication, polyurethane (PU) which has excellent flexibility, elasticity, good vibration resistance and high heat resistance was adopted as the matrix to fabricate the porous self-lubricating materials by NIPS and a series of PU films with different porosity were fabricated to obtain a porous self-lubricating PU film. The dependency between the porous structure and the preparation process is evaluated and the lubrication mechanism of the self-lubricating materials was discussed.

\section{Experimental}

\subsection{Materials}

Thermoplastic polyurethane (TPU, Elastollan C95A) which was derived from 4,4'-diphenylmethane diisocyanate, 1,4-butanediol and adipic acid was purchased from BASF, Germany. Methyl silicone oil (Density $=0.963 \mathrm{~g} / \mathrm{cm}^{3}$, Dynamic viscosity $\eta=$ $0.482 \mathrm{~Pa} \cdot \mathrm{s}$ ) using as lubricating agent was provided by Sichuan ZhongHao ChenGuang Institute of Chemical Research. $N, N$-Dimethylformamide (DMF) was obtained from Kaitong Chemical Reagent Co., Ltd, Tianjin, China. All the chemicals were used without further purification. 


\subsection{Preparation of the porous $P U$ materials with NIPS method}

Under the conditions of stirring speed and heating temperature of $100 \mathrm{rpm}$ and $80^{\circ} \mathrm{C}$, respectively. The TPU particles were dissolved in DMF to prepare homogeneous TPU solutions of different weights concentrations $(15,20,25$ and $30 \mathrm{wt} \%)$. Subsequently, the TPU solution was placed in a vacuum vessel of $-0.8 \mathrm{MPa}$ for 30 minutes to remove all bubbles in the solution. The degassed TPU solution was cast on clean a glass mold (100 mm length, $25 \mathrm{~mm}$ width and $0.6 \mathrm{~mm}$ depth) and immediately immersed into the coagulation bath with $60 \mathrm{wt} \% \mathrm{DMF}$ at ambient temperature after the excess TPU solution was removed with a spatula. After 4 hours, the formed film was soaked in deionized water to remove any traces of residual DMF solvent and subsequently air-dried at room temperature to remove residual water.

The porous PU films were dried at $80^{\circ} \mathrm{C}$ in vacuo for $2 \mathrm{~h}$, and immersed in lubricating oil under vacuum at $80^{\circ} \mathrm{C}$ for $10 \mathrm{~h}$ to make sure that the internal pores were maximally infiltrated. The lubricant on the surface was wiped off by acetone cotton to obtain the porous self-lubricating films, the weight of the samples before and after immersion was recorded to obtain the oil content.

\subsection{The oil retention test of porous self-lubricating PU materials}

The centrifugation test and heating test were carried out in order to statically simulate whether centrifugation and thermal effects during the friction process are able to extract lubricating oil from an impregnated porous membrane and evaluate the oil supply behavior of the PU throughout the sliding process. In centrifugation tests, the oil retention of porous PU oil-containing materials was measured by bench-top high-speed centrifuge (Changsha Xiangyi centrifuge Instrument Co., Ltd, TDZ5-WS type). Firstly, the lubricating oil emerging on the surface of the porous film was removed after centrifugation at the speed of $4000 \mathrm{r} / \mathrm{min}$ for $1 \mathrm{~min}$, and then the weight of each film was measured at intervals of $30 \mathrm{~min}$. In the measurement of heating, the porous PU oil-containing materials were placed on a self-made heating table. The temperature of the heating procedure rises from 30 to $150{ }^{\circ} \mathrm{C}$ at intervals of $30^{\circ} \mathrm{C}$, the weight of each sample was measured after holding $30 \mathrm{~min}$ at each temperature point. During the quantitative evaluating experiments, the spilled oil on the surface was wiped off by acetone cotton. Three samples were conducted under identical conditions for different porosity film, and the final results were taken as the average value. The oil content and oil retention were calculated by Equations (1) and (2) [21]:

Oil content $=\frac{\left(M_{1}-M_{0}\right) \rho_{0}}{M_{0} \rho_{1}} \cdot 100 \%$

Oil retention $=\frac{\left(M_{\mathrm{t}}-M_{0}\right) \rho_{0}}{M_{0} \rho_{1}} \cdot 100 \%$

where $M_{0}$ is the initial mass of the porous films with no oil immersion, $M_{1}$ is the mass of the porous films after being immersed into oil, $M_{\mathrm{t}}$ is the mass of the porous films after centrifugation or heating for $t \mathrm{~min}$ utes, $\rho_{0}$ is the bulk density of the porous films and measured by mercury injection apparatus. $\rho_{1}$ is the density of the lubricant oil.

\subsection{Friction and wear tests}

Tribological properties of the porous self-lubricating PU materials were investigated by block-on-ring wear tester (MRH-3, Jinan Yihua Tribology Testing Techonology Co., Ltd, China). The counterpart was a GCr 15 steel ring (ø $49.22 \mathrm{~mm}$ ), and the porous PU materials were cut into rectangular samples $(19 \mathrm{~mm} \times 12 \mathrm{~mm})$. The upper surface of the oil-containing self-lubricating films is adhered to the steel block by using an adhesive to form a friction sample. According to standard of GB3960-83, the friction coefficient of the porous self-lubricating materials was measured under the condition of fixed load of $200 \mathrm{~N}$ and rotational speed of $200 \mathrm{rpm}$, the duration time lasted for 120 minutes with a total sliding distance of 3709 meters.

To fully understand the lubrication mechanism of our materials, a contrast experiment was conducted. In this contrast trial, the friction couple along with porous PU materials without vacuum oil absorption procedure are fully immersed in an installation filled with lubricating oil which the regime of the friction could be transformed from full fluid lubrication to the boundary lubrication by varying the rotational speed between $5 \mathrm{rpm}$ (mean linear velocity of $0.01 \mathrm{~m} / \mathrm{s}$ ) and $760 \mathrm{rpm}$ (mean linear velocity of $1.96 \mathrm{~m} / \mathrm{s}$ ) under a fixed load of $200 \mathrm{~N}$. In other words, the untreated porous material was evaluated with the external oil supply. By comparing the friction status, 
the friction regime of our materials falls in our testing conditions could be illustrated and the lubrication mechanism in our trials could be elucidated.

\subsection{Characterization of porous self-lubricating PU materials}

The cryogenic-fractured cross-section morphology of the films were observed on a scanning electron microscope (SEM, JSM-5600LV, Japan) under an accelerating voltage of $20 \mathrm{kV}$ after being sputtered with gold. The pore size distribution and porosity of the porous PU films were evaluated by mercury injection apparatus which was performed on Micromeritics AutoPore IV9510 at ambient temperature. The three-dimensional topography of the worn surface was observed by optical surface profilometer MicroXAM-800 (KLA-tencor Inc., USA).

\section{Results and discussion}

\subsection{Microstructure and porosity of porous self-lubricating PU materials}

As mentioned above, the PU porous films were fabricated by non-solvent induced phase separation (NIPS), a relatively continuous, uniform porous structure could be formed by coagulation bath with $60 \mathrm{wt} \%$ DMF instead of distilled water, which contributed to the appropriate rate of exchange between the solvent and the nonsolvent in this case. The longterm phase change provides a sufficient time for the

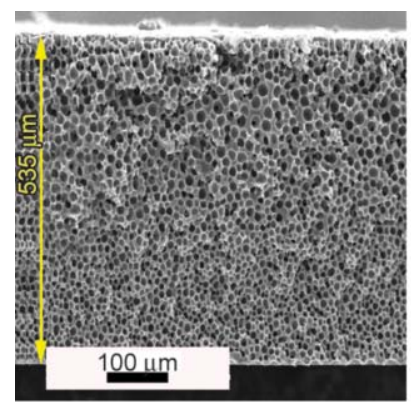

a)

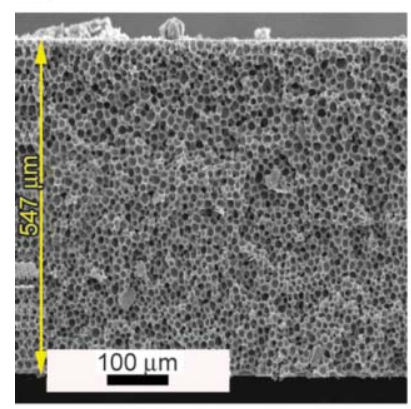

e)

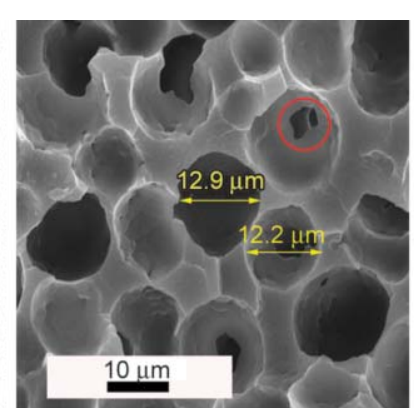

b)

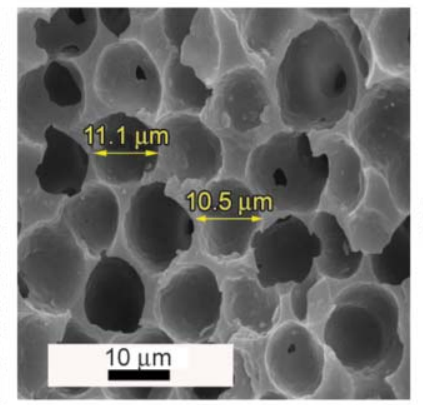

f) growth of the polymer-poor phase domain and the formation of pore well-distributed skeleton. The microstructure of porous PU films which prepared from different concentration is shown in Figure 1. It can be seen from Figure 1 that the internal pore structure is similar to that of a sponge, and as PU concentration increased, the size of pores decreased because the increased viscosity of PU reduced the phase separation degree, restrained the growth rate of pore. In addition, the thickness of film is about $546 \pm 11 \mu \mathrm{m}$ in the SEM image. Meanwhile, there are small holes (marked by red circle) in the inner wall of the pores by which the micrometer pores were connected to each other.

Mercury intrusion porosimetry was used to evaluate the pore size distribution of the PU porous films which were prepared from different concentrations solutions. And the results are demonstrated in Figure 2a, the sharp and narrow peaks appear at 1.61, 1.62, 2.09 and $2.53 \mu \mathrm{m}$, respectively. The pore size measured by mercury intrusion porosimetry is inconsistent with the size of the macropore observed in the SEM image. As observed from SEM, the macropores were interpenetrating with each other and the size value obtained from mercury intrusion porosimetry tests is corresponding to the size of the bottleneck pores in the porous films. This structure of pore can be further illustrated by the mercury intrusion curve in Figure $2 \mathrm{~b}$ which demonstrates that after completion of

Figure 1. Cross-section morphology of the PU porous films prepared by NIPS. The PU solution concentrations were as follows: (a, b) $15 \mathrm{wt} \%$, (c, d) $20 \mathrm{wt} \%$ (e, f) $25 \mathrm{wt} \%$ and (g, h) $30 \mathrm{wt} \%$. 

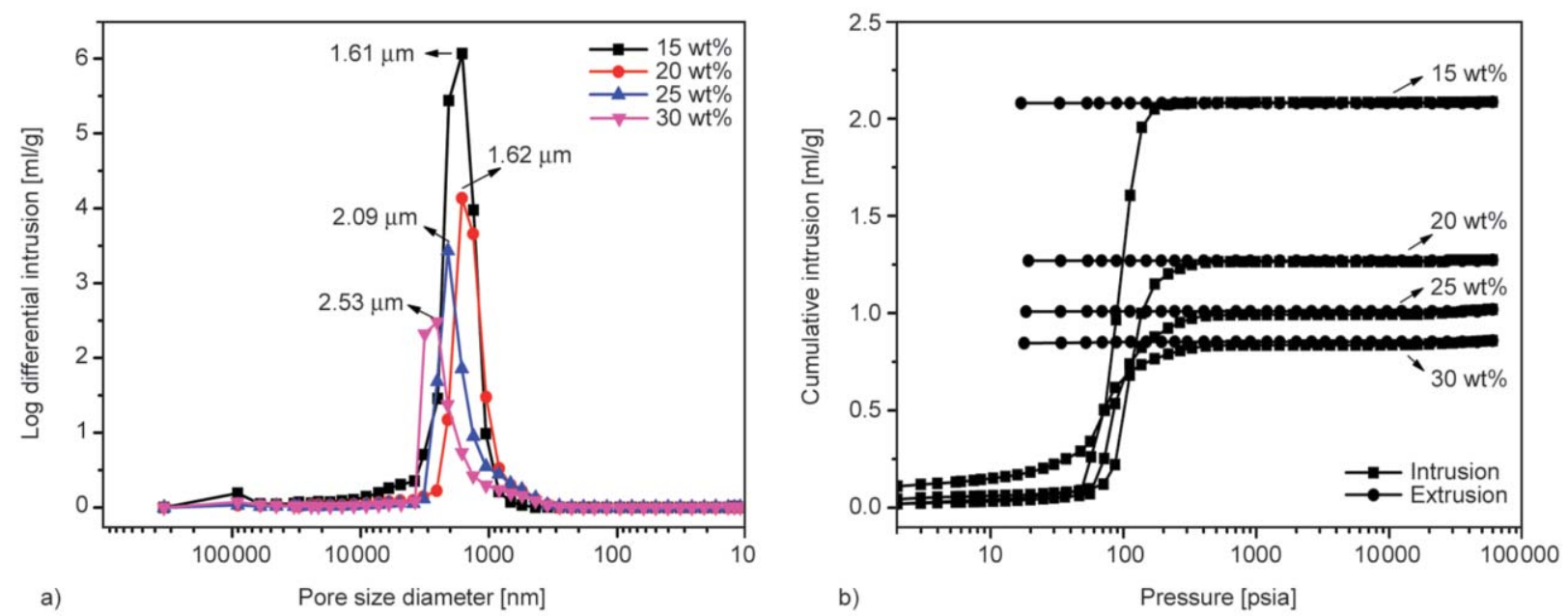

Figure 2. (a) The pore size distribution and (b) intrusion/extrusion branches of the porous PU films prepared from different PU solution concentrations.

mercury intrusion/extrusion cycle, not all intrude mercury is extruded from the sample during depressurization. The inability of retained mercury from being drawn back from the pore network, made intrusion curves are not retraceable. In other words, the small holes in materials prevent the mercury from leaking out in the procedure of extrusion. The hysteresis between intrusion and extrusion branch reveals the formation of 'ink-bottle'-type pores in porous PU films, which is consistent with the expectation by Wang et al. [22].

The data of the porosity of porous PU film prepared from different concentrations solutions are measured by mercury intrusion porosimetry listed in Table 1 . From Table 1, four samples with porosity of 69.93, $59.61,55.77$ and $51.26 \%$ were obtained with the concentration of PU solution increased from 15 to $30 \%$, which indicated that the increase of the concentration of PU solutions consequently decreases the porosity of the materials. The main reason could be attributed to the inhibition of molecular chain movement in higher PU concentration solution. In other words, the

Table 1. Relationship between PU solution concentration and film porosity, oil content, friction coefficient and wear depth (measured at the conditions of implemented load $200 \mathrm{~N}$ and rotational speed of $200 \mathrm{rpm}$ for $2 \mathrm{~h}$ ).

\begin{tabular}{|c|c|c|c|c|}
\hline $\begin{array}{c}\text { PU solution } \\
\text { concentration } \\
{[\mathbf{w t} \%]}\end{array}$ & $\begin{array}{c}\text { Porosity } \\
{[\mathbf{v o l \% ]}}\end{array}$ & $\begin{array}{c}\text { Oil content } \\
{[\mathbf{v o l \% ]}}\end{array}$ & $\begin{array}{c}\text { Friction } \\
\text { coefficient }\end{array}$ & $\begin{array}{c}\text { Wear } \\
\text { depth } \\
{[\boldsymbol{\mu} \mathbf{m}]}\end{array}$ \\
\hline 15 & 69.93 & 67.15 & 0.078 & 13.1 \\
\hline 20 & 59.61 & 59.32 & 0.121 & 15.5 \\
\hline 25 & 55.77 & 54.76 & 0.135 & 22.3 \\
\hline 30 & 51.26 & 45.78 & 0.174 & 31.4 \\
\hline
\end{tabular}

low PU concentration facilitated folding of the PU molecule during the process of phase separation.

Combining the results of SEM and mercury intrusion porosimetry, it indicated that the cross-section morphology of the films is similar to that of the sponge and the film has an 'ink-bottle' type pore structure. Meanwhile, as the PU solution concentration increased from 15 to $30 \%$, the porosity of the four samples ranged from 69.93 to $51.26 \%$ and the size of the bottleneck pores in the porous membrane increased from 1.61 to $2.53 \mu \mathrm{m}$. Put it another way, the porosity of the porous PU films can be controlled by varying the PU solution concentrations in the DMF solution.

\subsection{Oil-retention of porous self-lubricating PU materials}

Figure 3a shows the variation of oil retention with the different centrifugal time for four PU oil-containing materials after $4000 \mathrm{r} / \mathrm{min}$ rotation. For the sample with $69.93 \%$ porosity, the average value of oil content was found to be $67.15 \%$ after being immersed into oil. When the porosities were 59.61, 55.77 and $51.26 \%$, the oil content of the porous materials dropped down to 59.32, 54.76 and $45.78 \%$, respectively. After 150 minutes of centrifugation, $78.98,81.95,83.48$ and $93.10 \%$ of the oil retention values for the four samples with different porosity were demonstrated compared to the original oil content. Based on the fact that the structure of porous film is ink-bottle type pores, the resistance caused by Laplace pressure can partially resist the centrifugal force. Therefore, a large amount of lubricant cannot be extracted from the pores. Consequently, the 

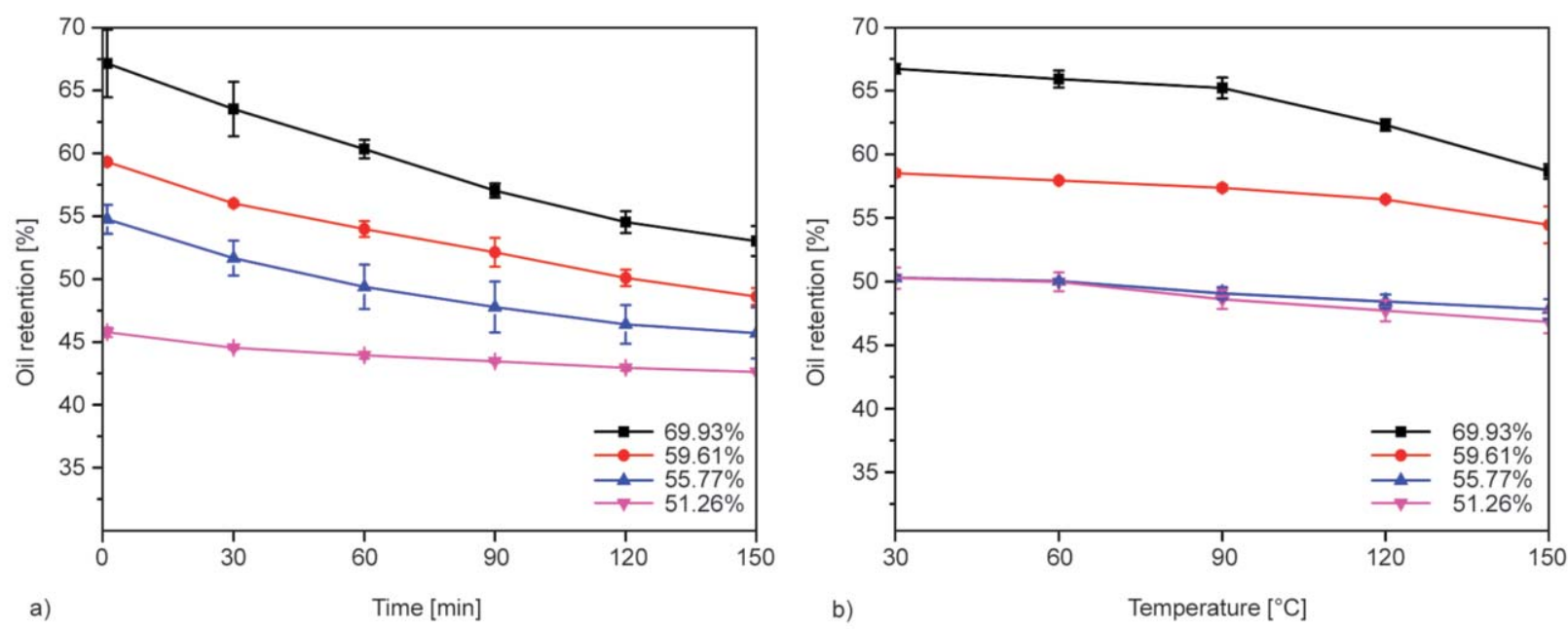

Figure 3. Oil retention of porous materials with different porosity (a) variation of oil retention with centrifugal time at ambient temperature and pressure; (b) variation of oil retention with temperature at ambient pressure.

porous film still has high oil content under centrifugal action, which exhibited it excellent oil retention performance.

The effect of temperature on the oil retention of the porous film is exhibited in Figure 3b. In general, the higher the temperature is, the more the oil retention decreases. At a given temperature, the static pressure generated by the liquid column is equal to the Laplace force of the oil $(P=2 \gamma / r=\rho g h)$, so that the height $(h)$ of the liquid column in the pore is constant. The increase of temperature reduces the surface tension $(\gamma)$ of lubricating oil which subsequently decreased Laplace pressure. In other words, a decrease in the height $(h)$ of the liquid column means that the lubricating oil will overflow from the pores. Meanwhile, as lubricating oil is heated, the increase of molecular motion results in the increase of intermolecular spacing and finally an increase in the volume of the lubricating oil. These two factors both contribute to the overflowing of the lubricating oil from the macropores. It is also noticeable that the oil retention of the materials with porosity of 55.77 and $51.26 \%$ are similar, which could attribute to the proximity of the bottleneck pore size and oil content. Combining the centrifugal and heating results, temperature variation has less impact on the oil retention properties of the PU materials compared with the centrifugal action.

\subsection{Tribological properties of porous self-lubricating PU materials}

Figure 4 shows the friction coefficient curves of porous self-lubricating PU materials with different porosity under $200 \mathrm{rpm}$ and fixed load of $200 \mathrm{~N}$ for
$2 \mathrm{~h}$ at ambient temperature. It can be observed that the friction curve of pure sample (by nomenclature, the sample with a porosity of $51.26 \%$ and without immersion in lubricating oil as pure sample) is unstable, and noise sustained during the whole friction process. This stick-slip vibration dominated the pure materials frictional behavior in a dry friction state. It was remarkable that the friction coefficient of pure sample decreased rapidly from 0.54 to 0.36 at $\sim 670$ seconds, i.e., the friction coefficient of the steel substrate, which is a sign of the worn-out failure for the pure sample. Besides, it can also be seen from the SEM of worn surface and counterpart surface in Figure 5a that the porous film tore up, quantities of debris appeared on the friction surface and thick transfer film appeared in Figure 5f, all of which illustrate

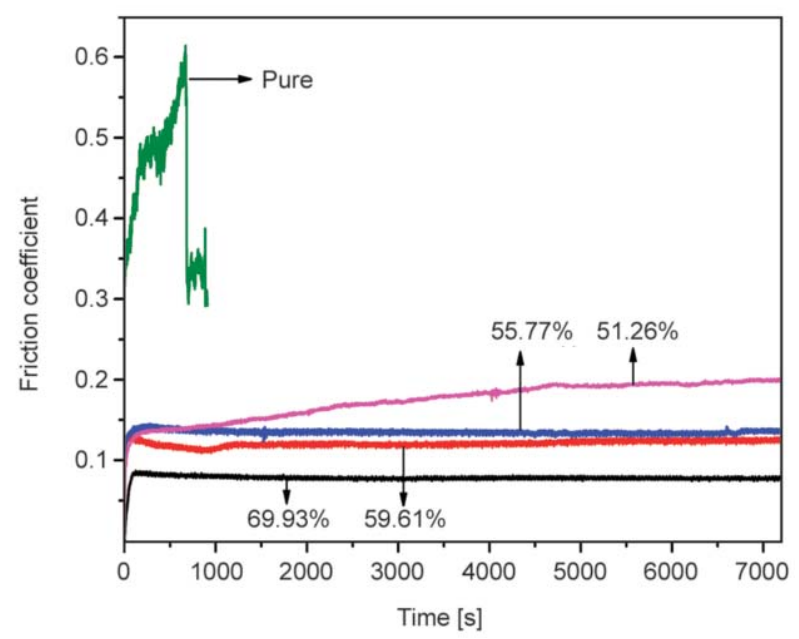

Figure 4. The friction coefficients of oil-containing samples with different porosity and pure sample under the conditions of implemented load $200 \mathrm{~N}$ and rotational speed of $200 \mathrm{rpm}$ for $2 \mathrm{~h}$. 
that the fatigue wear dominate in the friction of pure sample.

In the case of self-lubricating PU materials prepared by immersing porous film in lubrication oil, the formation of lubricating film having a certain bearing capacity between the friction surfaces to separate the two friction surfaces, which have less shear resistance when sliding, and resulting in lower friction coefficient. Therefore, the average friction coefficient of porous self-lubricating PU materials with porosity of $69.63,59.61$ and $55.77 \%$ were $0.078,0.121$, and 0.135 , respectively, and the friction coefficient curve tends to be smooth and stable during the whole sliding test as demonstrated in Figure 4. Figures 5b, 5c, $5 \mathrm{~d}$ shows SEM images of the worn surface of the corresponding samples, the scale protuberance which is a sign of plastic deformation and plastic flow along the friction direction appeared, especially for samples with porosity of 59.61 and $55.77 \%$. Besides, there is no debris on the counterpart surface (shown in Figures 5g, 5h, 5i) for all the samples. Two contradicting factors will account for the phenomena: on the one hand, the lower solid content in the DMF solution contributed to higher porosity further higher oil content in the sample which will favor the reduction of friction force. On the other hand, the higher porosity of the sample gives rise to the reduction of the anti-shear ability of matrix which made the material prone to be arranged parallel to the shear force during the sliding. Ultimately, the latter factor dominated in the friction thus the as-observed morphology could be observed. For a porous self-lubricating PU material with a porosity of $51.26 \%$, the friction coefficient gradually increases and finally becomes stable with the increase of time. It can be seen from the SEM image of worn surface (shown in Figure 5e) that the material has relatively smooth wear could be observed with the pore structure remained in the worn surface.

The three-dimensional optical surface profilometer was applied to intuitively investigate the topography of worn surface and results were presented in Figure 6 . In the case of samples with porosity of 69.93 and $59.61 \%$, it can be seen that the wear scar of the materials was shallow and the wear depth increased from 13.1 to $15.5 \mu \mathrm{m}$ in Figures $6 \mathrm{a}, 6 \mathrm{~b}$. For a porous self-lubricating PU material with porosity of $55.77 \%$, the worn surface is seen in Figure $6 \mathrm{c}$ as roughness and the depth of wear reaches $22.3 \mu \mathrm{m}$. In this situation, this material does not show excellent wear resistance even though the values of friction coefficient are still stable compared to other samples. The reason is most probably that the lubricating oil can completely fill rugged surface to form effective lubricating film. In the case of samples with porosity of $51.26 \%$, we can clearly see that the wear surface of the sample is relatively poor and the wear depth is $31.4 \mu \mathrm{m}$. Meanwhile, it is obviously observed in Figure $6 \mathrm{~d}$ that there are fewer pores on the surface of the sample, which caused the decrease of lubricating oil extruded and consequently failed to form effective lubricating film. According to the above experimental results, it can be concluded that the friction coefficient and wear depth is decreased with increase in porosity in these porous self-lubricating PU materials. Therefore, the tribological properties of porous self-lubrication materials were closely associated with the structure of the materials.

In order to further investigate the influence of load and rotational speed on friction coefficient at room temperature and ambient humidity, we selected samples with porosity of $69.93 \%$ as the testing specimens because of its lowest friction coefficient and wear. In Figure 7a, the five loads of the block-onring wear tester are set to $50,150,250,350$ and $450 \mathrm{~N}$ with the rotational speed fixed at $200 \mathrm{rpm}$, it is apparent that the load has a significant effect on friction coefficient of porous self-lubricating films. Under the load of $50 \mathrm{~N}$, the average friction coefficient of the material is 0.242 and the fluctuation of friction coefficient is more obvious than other curves. It is evident that the friction coefficient of porous self-lubricating materials becomes progressively lower with the increasing load. When the load finally reaches $450 \mathrm{~N}$, the average friction coefficient of the material reaches the lowest (0.045), which exhibited relatively excellent tribological properties during the entire sliding. The lubrication mechanism of the porous self-lubricating materials is similar to the joint lubrication mechanism when the joint is subjected to a normal load, the lubricant overflows from the pores of cartilage tissue to provide lubrication. Similarly, the higher load applied on the porous film, the more lubricating oil will be squeezed to form an effective lubricating film. Moreover, heat is inevitably generated between the friction interfaces during the sliding process. Previous experiments have illustrated that the thermal effect had a certain influence on the release of lubricating oil (previously shown in Figure 3). However, Figure 7b demonstrates that the 
a)

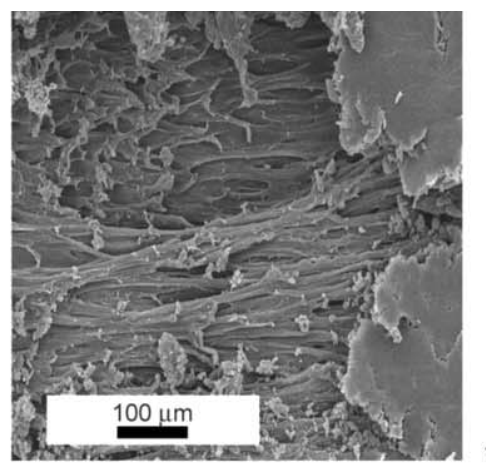

b)

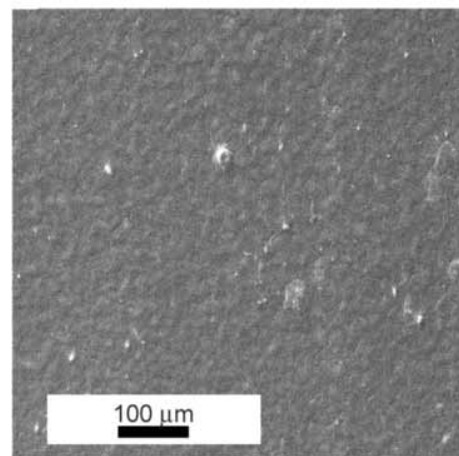

c)
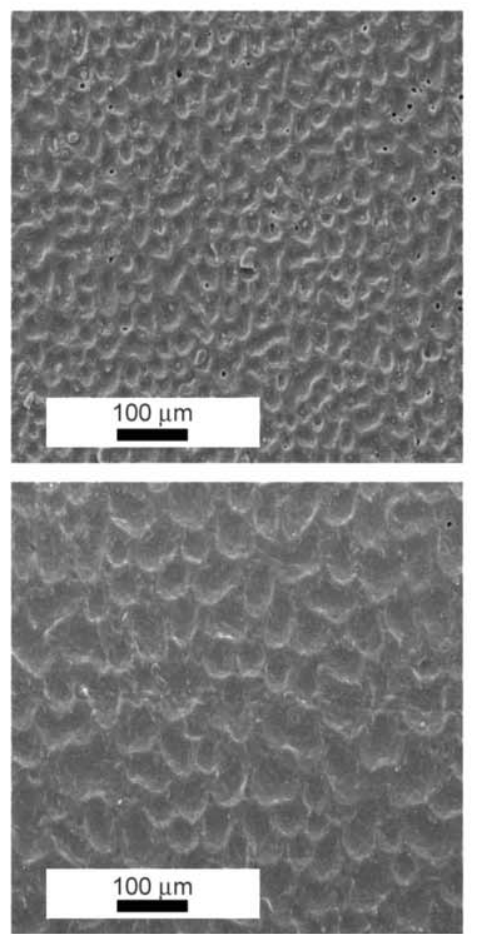

d)

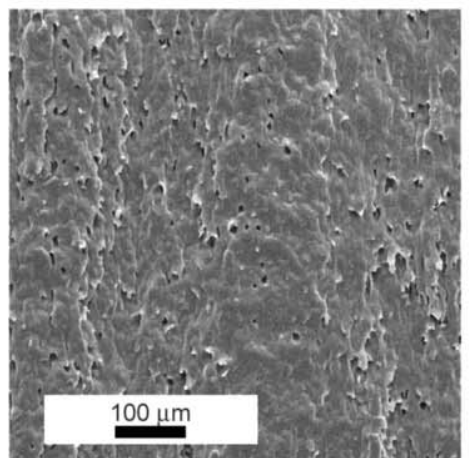

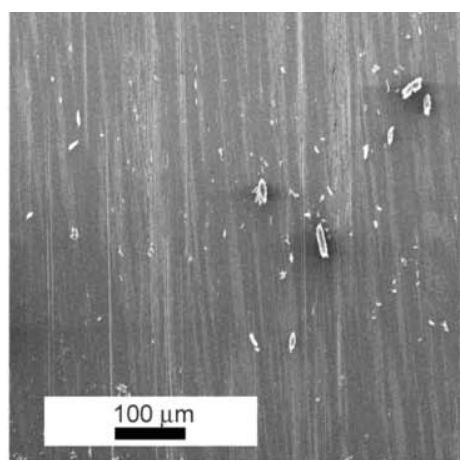
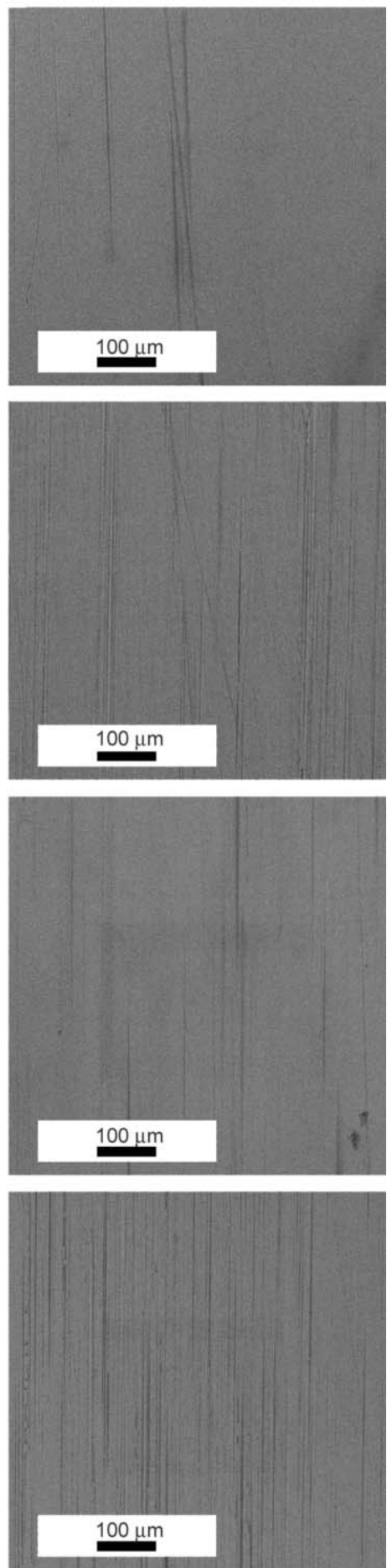

Figure 5. SEM images of worn surfaces and wear-track on steel ring. Pure sample (a, f), the porosity of oil-containing samples are as follows: (b, g) $69.93 \%,(c, h) 59.61 \%$, (d, i) $55.77 \%,(\mathrm{e}, \mathrm{j}) 51.26 \%$. 

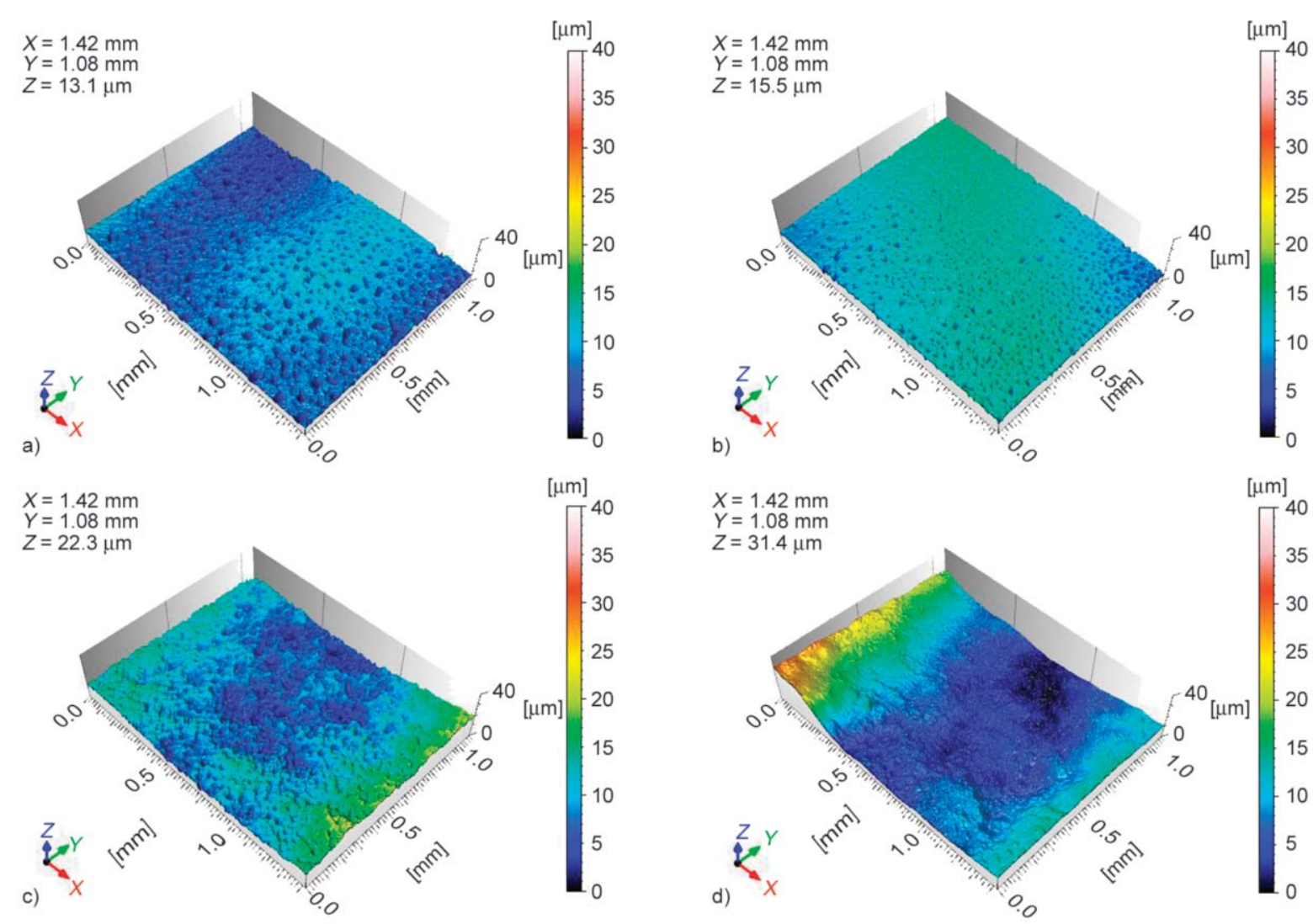

Figure 6. Three-dimensional morphologies of worn surface of self-lubricating materials with different porosity. (a) $69.93 \%$; (b) $59.61 \%$; (c) $55.77 \%$; (d) $51.26 \%$.

maximum temperature during friction is only $\sim 38^{\circ} \mathrm{C}$. In other words, the temperature generated by thermal effects was unable to extract enough lubricating oil from porous materials. The above results allow us to infer that the applied normal load dominating the release of lubricating oil further the frictional and wear behavior other than the temperature rising in the scope of our testing conditions.

Figure $7 \mathrm{c}$ clearly indicates that there is a raise in friction coefficient with an increase in rotational speeds under the load fixed at $200 \mathrm{~N}$, the average coefficient of friction of the materials was $0.058,0.069,0.085$, 0.089 and 0.122 at 50, 150, 250, 350 and $450 \mathrm{rpm}$, respectively. Meanwhile, Figure $7 \mathrm{~d}$ clearly presented that the frictional heat is greatly affected by the rotational speed during wear test. Compared with the results of effect of load on temperature (Figure 7b), the higher rotational speed applied, the evident increase of temperature displayed.

It is well known that the variation of friction coefficient with the presence of external lubricant is strongly dependent on U $\eta$ which was systematically studied by Stribeck, where $U$ is the sliding velocity and $\eta$ is the dynamic viscosity of the lubricant [23]. According to the classical Stribeck theory, the lubrication regimes for liquid-lubricated surfaces were categorised into three regimes: boundary lubrication, mixed lubrication and fluid lubrication. In the boundary lubrication regime, the thickness of the lubrication film between the friction surfaces is only one or two monolayers, so the frictional resistance was basically determined by the solid friction between the friction pairs. In the fluid lubrication regime, the surface of the friction pair was isolated by the fluid lubricating film, and the frictional resistance was only caused by the internal friction of the fluid. However, in the mixed lubrication, the frictional resistance was caused by the internal friction of the lubricating fluid and the solid friction between the friction pairs. In order to determine the lubrication mechanism of the as-fabricated PU porous materials, the friction coefficient of sample (with porosity of $69.93 \%$ ) without oil vacuum immersion was evaluated under the full immersion of liquid lubricant. Figure 8 shows the tendency of measured friction coefficient versus $U \eta$, it can be found that the plot conformed to the Stribeck curve. The boundary lubrication regime occurs before the value of friction coefficient is 0.96 , and subsequently the frictional curve begins to decrease with the increase of the $U \eta$ 

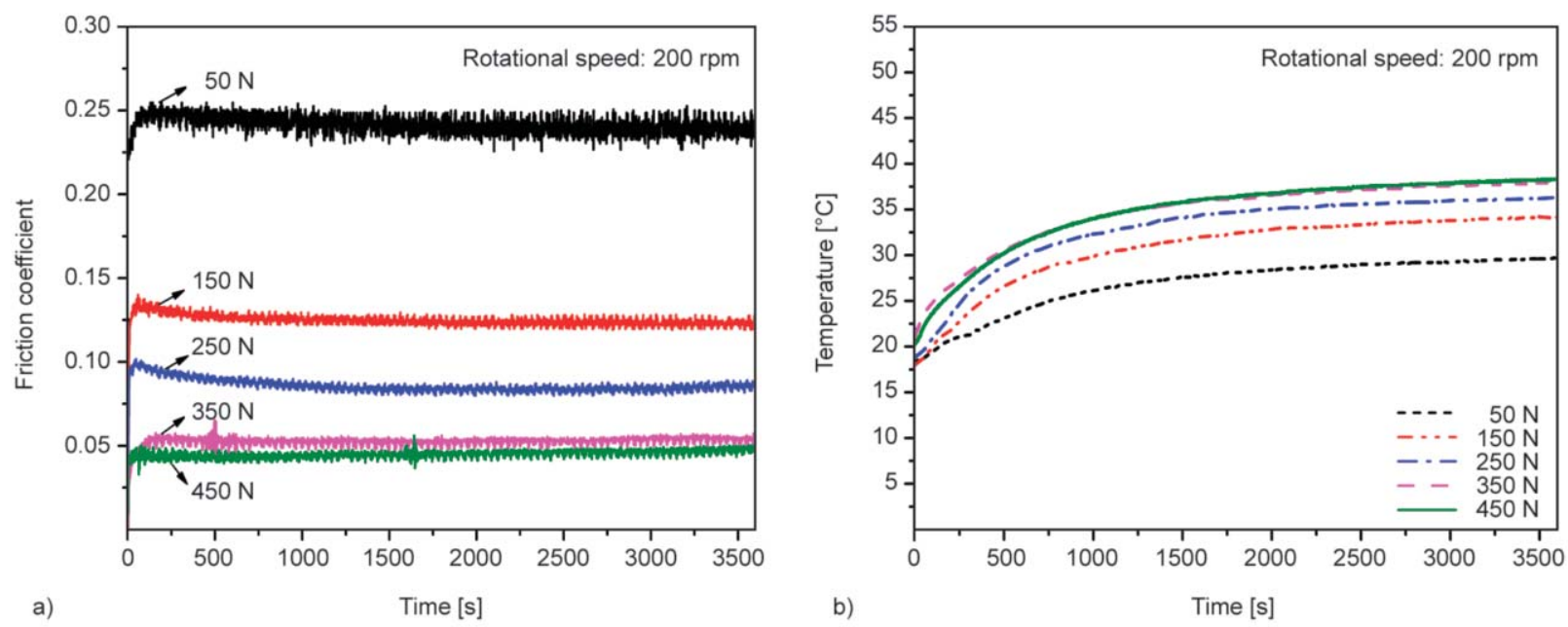

a)
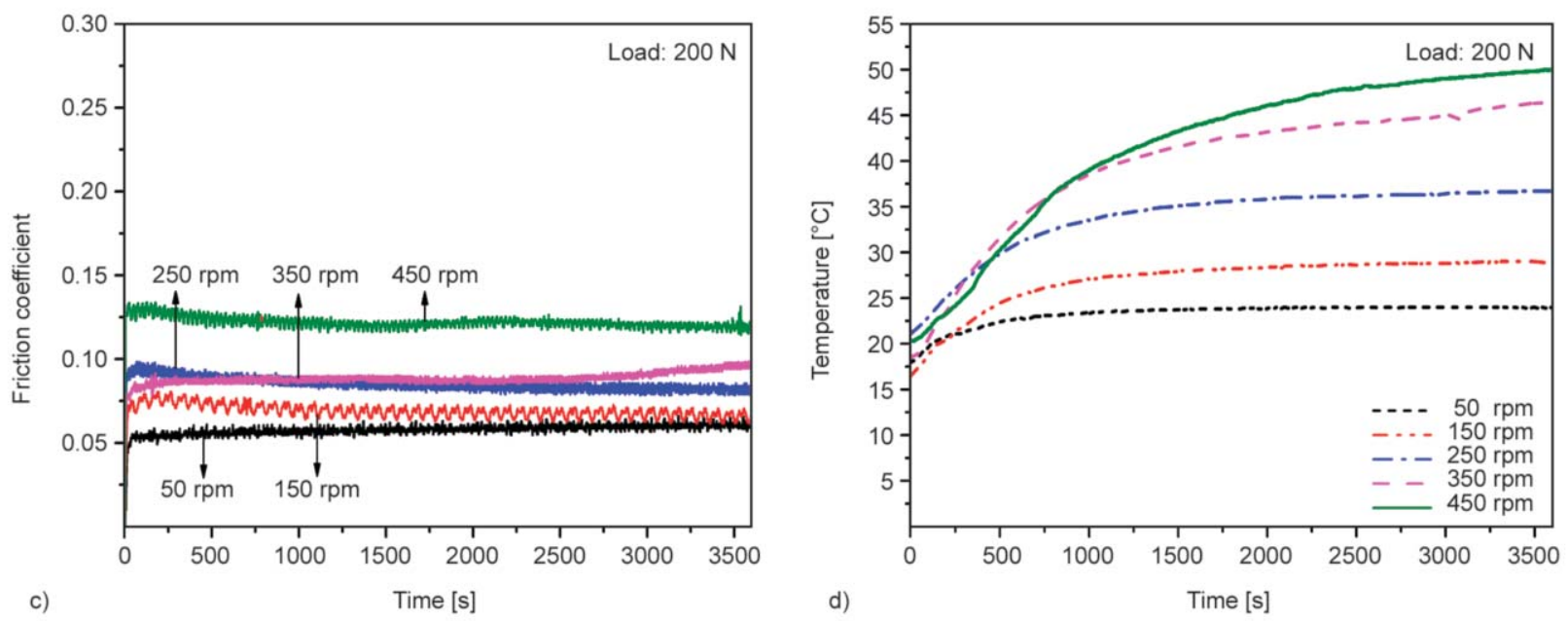

Figure 7. Tribological properties of porous self-lubricating materials. (a) Friction coefficient of self-lubricating materials versus different loads; (b) Temperature variation of self-lubricating materials in friction process versus different loads; (c) Friction coefficient of self-lubricating materials versus different speeds; (d) Temperature variation of sample in friction process versus different speeds.

which corresponds to the mixed lubrication regime. Besides, the fluid lubrication is achieved after the friction coefficient reaches the values of 0.02 . The Stribeck curve exhibited that the friction coefficient increases with the increase of the $U \eta$ during the fluid lubrication regime. At first glance, the friction coefficient obtained in the friction test without immersing the friction device into the lubricating oil (shown in Figure 7c) increases with the increase of the rotational speed, which should belong to the fluid lubrication regime, but all values of the friction coefficients in Figure $7 \mathrm{c}$ are higher than those in the fluid lubrication regime and fall in the boundary or mixed lubrication regime. As we know, under mixed lubrication conditions, the frictional force between two solid surfaces in the presence of fluid can be divided into two parts: friction between solid surface $f_{\mathrm{s}}$ which is mainly originated from the contact mechanics of asperities from two solid surfaces; the internal

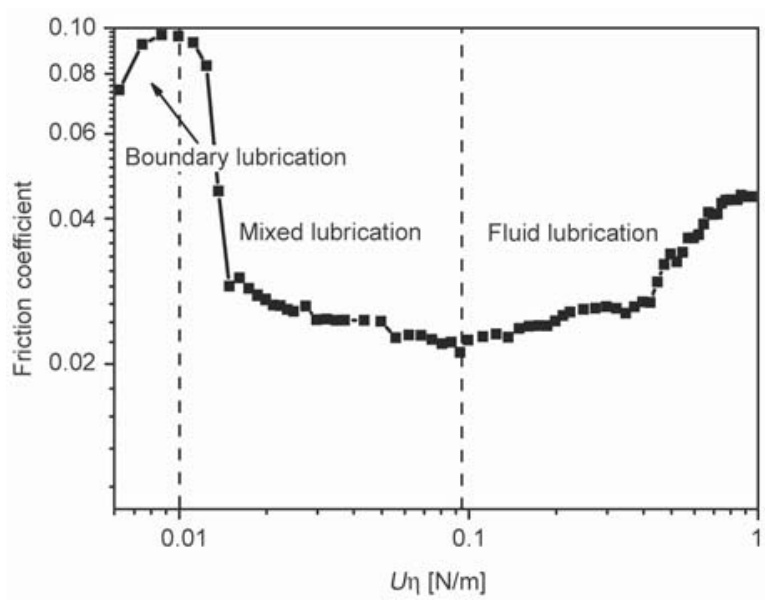

Figure 8. Friction coefficient as function of $U \eta$ at the load of $200 \mathrm{~N}$.

shear force due to the lubricating fluid $f_{\mathrm{L}}$ which is equal to the product of viscosity and velocity gradient. With the increase of velocity gradient, the $f_{\mathrm{L}}$ increases accompanied the thickness of the lubricating 
film increase as well. In the meanwhile, the $f_{\mathrm{s}}$ decreases. On the whole, the frictional force decreases with the increase of velocity. From this point of view, the lubrication mechanism for our sample in the testing conditions is boundary lubrication. Actually, the oil content of our sample is so high that the oil is prone to be squeezed out of the sample. Thus, if the squeezed oil could persist and stabilize to form the lubricating film between the gap of the friction couple, the lubrication is mixed lubrication or even fluid lubrication. However, in our system here, the load bearing capability of the porous material could not withstand the dynamic pressure generated by the fluid film oozes from the porous materials. Therefore, the squeezed oil could be re-infiltrated back into the pores of the materials or be forced to emigrate from the friction zone which could be observed in the friction trials as well. Thus, it can be reasonably inferred that the lubricating oil contained in the self-lubricating porous material can only maintain the material in boundary lubrication regime rather than mixed lubrication regime during friction process.

\section{Conclusions}

The porous polyurethane films with controlled porosity and uniform contiguous pores were prepared by non-solvent induced phase separation (NIPS) method. Particularly, the porous film is capable of inhaling and storing lubricating oil because of ink-bottle type pores. Therefore, the porous films still have high oil retention under the influence of centrifugation and thermal effects. Compared with the neat porous PU films, the oil-containing porous films exhibit excellent tribological properties, thanks to its high oil content. Simultaneously, variations in friction coefficient depend on the release of lubricating oil and formation of lubrication film which was further governed by the load and rotational speed. It is found that the friction coefficient is 0.058 at $50 \mathrm{rpm}$ speed and 0.045 at $450 \mathrm{~N}$ load. Consequently, based on its simple manufacture process of the porous self-lubricating PU materials via NIPS, the as-prepared selflubricating materials may possess significant prospects for tribological applications

\section{Acknowledgements}

This research was financially supported by National Natural Science Foundation of China (51465036) and Provincial Youth Science and Technology Foundation of Gansu (1506RJYA089).

\section{References}

[1] Itoigawa F., Nakamura T., Matsubara T.: Starvation in ball bearing lubricated by oil and air lubrication system. Tribology, 34, 243-252 (1998). https://doi.org/10.1016/S0167-8922(98)80079-5

[2] Mu B., Li X., Yang B., Cui J., Wang X., Guo J., Bao X., Chen L.: Tribological behaviors of polyurethane composites containing self-lubricating microcapsules and reinforced by short carbon fibers. Journal of Applied Polymer Science, 134, 45331/1-45331/10 (2017). https://doi.org/10.1002/app.45331

[3] Yang M., Zhu X., Ren G., Men X., Guo F., Li P., Zhang Z.: Tribological behaviors of polyurethane composite coatings filled with ionic liquid core/silica gel shell microcapsules. Tribology Letters, 58, 9/1-9/9 (2015). https://doi.org/10.1007/s11249-015-0492-7

[4] Wang J., Zhao H., Huang W., Wang X.: Investigation of porous polyimide lubricant retainers to improve the performance of rolling bearings under conditions of starved lubrication. Wear, 380, 52-58 (2017).

https://doi.org/10.1016/j.wear.2017.03.008

[5] Wang H., Li M., Liu D., Zhao Y., Zhu Y.: Tribological properties tests and simulations of the nano-micro multilevel porous self-lubricating PEEK composites with ionic liquid lubrication. Journal of Materials Science, 51, 3917-3927 (2016). https://doi.org/10.1007/s10853-015-9711-8

[6] Marchetti M., Meurisse M-H., Vergne P., Sicre J., Durand M.: Analysis of oil supply phenomena by sintered porous reservoirs. Tribology Letters, 10, 163-170 (2001). https://doi.org/10.1023/A:1009026123907

[7] Ruggiero A., Gòmez E., D’Amato R.: Approximate analytical model for the squeeze-film lubrication of the human ankle joint with synovial fluid filtrated by articular cartilage. Tribology Letters, 41, 337-343 (2011). https://doi.org/10.1007/s11249-010-9710-5

[8] Hui A. Y., McCarty W. J., Masuda K., Firestein G. S., Sah R. L.: A systems biology approach to synovial joint lubrication in health, injury, and disease. Wires Systems Biology and Medicine, 4, 15-37 (2011). https://doi.org/10.1002/wsbm.157

[9] Wang Q., Wang C., Wang T.: Controllable low dielectric porous polyimide films templated by silica microspheres: Microstructure, formation mechanism, and properties. Journal of Colloid and Interface Science, 389, 99-105 (2013).

https://doi.org/10.1016/j.jcis.2012.08.036 
[10] Chang C-J., Tsai M-H., Chen G-S., Wu M-S., Hung T-W.: Preparation and properties of porous polyimide films with $\mathrm{TiO}_{2} /$ polymer double shell hollow spheres. Thin Solid Films, 517, 4966-4969 (2009).

https://doi.org/10.1016/j.tsf.2009.03.201

[11] Lu J., Tang H., Xu C., Jiang S.: Nafion membranes with ordered mesoporous structure and high water retention properties for fuel cell applications. Journal of Materials Chemistry, 22, 5810-5819 (2012).

https://doi.org/10.1039/C2JM14838B

[12] Ye Q., Xiang Y., Chen F., Xu W., Yang H.: The formation of regular porous polyurethane membrane via phase separation induced by water droplets from ultrasonic atomizer. Materials Letters, 100, 23-25 (2013). https://doi.org/10.1016/j.matlet.2013.02.096

[13] Xing Q., Dong X., Li R., Yang H., Han C. C., Wang D.: Morphology and performance control of PLLA-based porous membranes by phase separation. Polymer, 54, 5965-5973 (2013).

https://doi.org/10.1016/j.polymer.2013.08.007

[14] Yang H., Ye Q., Zhou Y., Xiang Y., Xing Q., Dong X., Wang D., Xu W.: Formation, morphology and control of high-performance biomedical polyurethane porous membranes by water micro-droplet induced phase inversion. Polymer, 55, 5500-5508 (2014).

https://doi.org/10.1016/j.polymer.2014.08.058

[15] Abdel-Karim A., Gad-Allah T. A., El-Kalliny A. S., Ahmed S. A., Souaya E. R., Badawy M. I., Ulbricht M.: Fabrication of modified polyethersulfone membranes for wastewater treatment by submerged membrane bioreactor. Separation and Purification Technology, 175, 36-46 (2017). https://doi.org/10.1016/j.seppur.2016.10.060

[16] Ji D., Xiao C., An S., Liu H., Chen K., Hao J., Zhang T.: Preparation of PSF/FEP mixed matrix membrane with super hydrophobic surface for efficient water-in-oil emulsion separation. RSC Advances, 8, 10097-10106 (2018).

https://doi.org/10.1039/C8RA00055G
[17] Zhang H., Lin C-E., Zhou M-Y., John A. E., Zhu B-K.: High thermal resistance polyimide separators prepared via soluble precusor and non-solvent induced phase separation process for lithium ion batteries. Electrochimica Acta, 187, 125-133 (2016).

https://doi.org/10.1016/j.electacta.2015.11.028

[18] Danesh E., Ghaffarian S. R., Molla-Abbasi P.: Non-solvent induced phase separation as a method for making high-performance chemiresistors based on conductive polymer nanocomposites. Sensors and Actuators B: Chemical, 155, 562-567 (2011).

https://doi.org/10.1016/j.snb.2011.01.008

[19] Jeon J. W., Han J. H., Kim S-K., Kim D-G., Kim Y. S., Suh D. H., Hong Y. T., Kim T-H., Kim B. G.: Intrinsically microporous polymer-based hierarchical nanostructuring of electrodes via nonsolvent-induced phase separation for high-performance supercapacitors. Journal of Materials Chemistry A, 6, 8909-8915 (2018). https://doi.org/10.1039/c8ta02451k

[20] Abzan N., Kharaziha M., Labbaf S., Saeidi N.: Modulation of the mechanical, physical and chemical properties of polyvinylidene fluoride scaffold via non-solvent induced phase separation process for nerve tissue engineering applications. European Polymer Journal, 104, 115-127 (2018). https://doi.org/10.1016/j.eurpolymj.2018.05.004

[21] Jia Z., Yan Y., Wang W.: Preparation and tribological properties of PI oil-bearing material with controllable pore size. Industrial Lubrication and Tribology, 69, 8894 (2017).

https://doi.org/10.1108/ILT-09-2015-0125

[22] Wang C., Wang Q., Wang T.: Simple method for preparation of porous polyimide film with an ordered surface based on in situ self-assembly of polyamic acid and silica microspheres. Langmuir, 26, 18357-18361 (2010). https://doi.org/10.1021/la103473u

[23] Woydt M., Wäsche R.: The history of the Stribeck curve and ball bearing steels: The role of Adolf Martens. Wear, 268, 1542-1546 (2010).

https://doi.org/10.1016/j.wear.2010.02.015 